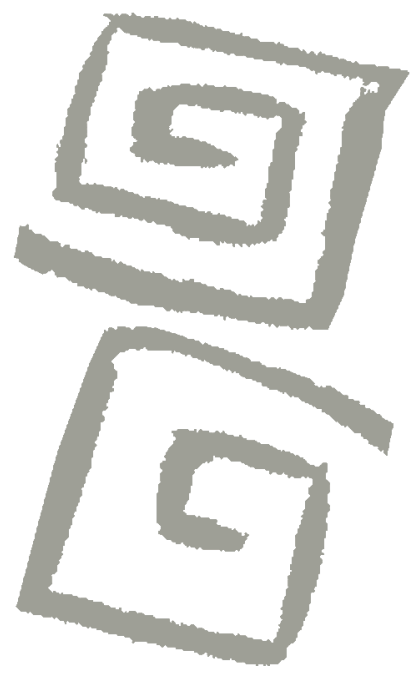

\title{
Prevalencia del síndrome de Down al nacimiento en Argentina
}

\section{Birth prevalence of Down syndrome in Argentina}

Javier Martini', María Paz Bidondo², Santiago Duarte³, Rosa Liascovich4, Pablo Barbero $^{5}$, Boris Groisman ${ }^{6}$

${ }^{1}$ Médico residente en Genética Médica. RENACCNGM, ANLIS Dr. Carlos G. Malbrán, Ministerio de Salud y Desarrollo Social Argentina. $\triangle$ (iD)

${ }^{2}$ Médica especialista en Genética Médica y Epidemiología. RENACCNGM, ANLIS Dr. Carlos G. Malbrán, Ministerio de Salud y Desarrollo Social, Argentina. $\triangle$ (iD

${ }^{3}$ Médico residente en Genética Médica. RENACCNGM, ANLIS Dr. Carlos G. Malbrán, Ministerio de Salud y Desarrollo Social Argentina. $\square$ (iD)

${ }^{4}$ Doctora en Ciencias Biológicas. RENAC-CNGM ANLIS Dr. Carlos C. Malbrán, Ministerio de Salud y Desarrollo Social, Argentina. $\triangle$ iD

${ }^{5}$ Médico especialista en Genética Médica, Doctor en Medicina. RENACCNGM, ANLIS Dr. Carlos G. Malbrán, Ministerio de Salud y Desarrollo Social, Argentina. $\triangle$ iD

${ }^{6}$ Médico especialista en Genética Médica Epidemiología. RENACCNGM, ANLIS Dr. Carlos G. Malbrán, Ministerio de Salud y Desarrollo Social Argentina. $\triangle$ iD
RESUMEN El objetivo de este trabajo fue describir la prevalencia al nacimiento del síndrome de Down en Argentina. Se calculó la prevalencia por jurisdicción y edad materna para el período 2009-2015 y se comparó la prevalencia y proporción del diagnóstico prenatal según subsector (público y privado) y nivel de complejidad de las maternidades. Se analizó la asociación con el peso y la edad gestacional al nacer. La fuente de datos fue la Red Nacional de Anomalías Congénitas (RENAC). La prevalencia fue de 17,26 por cada 10.000 nacimientos; por jurisdicciones varió entre 10,99 y 23,71, y por edad materna entre 10,32 en $<20$ años y 158,06 en $\geq 45$ años. En hospitales del subsector privado hubo una mayor proporción de diagnóstico prenatal y una mayor prevalencia, esta última atribuible a diferencias en la estructura de edad materna. Se observó una correlación negativa entre el peso al nacer y este síndrome $(\beta=-294,7 ; p<0,001)$. No se evidenció diferencia en la mediana de la edad gestacional al nacer entre recién nacidos con síndrome de Down y neonatos sin anomalías mayores, pero sí en la distribución de la edad gestacional. El conocimiento de ciertas características epidemiológicas podrá contribuir a la implementación de políticas de salud.

PALABRAS CLAVES Síndrome de Down; Epidemiología; Edad Materna; Argentina.

\begin{abstract}
The aim of this study was to describe the prevalence at birth of Down syndrome in Argentina. The prevalence by jurisdiction and maternal age was calculated for the 2009-2015 period and the prevalence and proportion of prenatal diagnosis was compared according to sub-sector (public and private) and complexity level of the maternity wards. The association of Down syndrome with birth weight and gestational age was analyzed. The data source was the National Network of Congenital Anomalies of Argentina [Red Nacional de Anomalías Congénitas] (RENAC). The prevalence was 17.26 per 10,000 births; by jurisdictions it varied between 10.99 and 23.71; and by maternal age, between 10.32 in women $<20$ years of age and 158.06 in those $\geq 45$ years of age. In hospitals of the private subsector there was a higher prevalence, attributable to differences in the structure of maternal age, and a greater proportion of prenatal diagnosis. There was a negative correlation between birth weight and Down syndrome $(\beta=-294.7 ; p<0.001)$. No difference in the median gestational age at birth between Down syndrome newborns and newborns without major anomalies was found, but the distribution of gestational age differed. Knowledge of certain epidemiological characteristics of this health issue could contribute to the implementation of health policies.
\end{abstract}

KEY WORDS Down Syndrome; Epidemiology; Maternal Age; Argentina. 


\section{INTRODUCCIÓN}

Las anomalías congénitas son aquellas alteraciones morfológicas o funcionales presentes desde el nacimiento. Su prevalencia en recién nacidos es del $3 \%$ al $5 \%{ }^{(1)}$. Con el control de las enfermedades infecciosas y nutricionales, las anomalías congénitas han incrementado su importancia relativa en la mortalidad infantil, pasando de representar el $11 \%$ en el año $1980^{(2)}$, al $27 \%$ en 2016 , siendo la segunda causa en la Argentina(3). Las causas conocidas de anomalías congénitas incluyen mutaciones en un gen principal, anomalías cromosómicas, la exposición prenatal a factores teratogénicos y el efecto de genes predisponentes que se expresan en presencia de factores ambientales desencadenantes. Tradicionalmente, se consideraba las anomalías congénitas como "no reducibles"; sin embargo, son múltiples las acciones de prevención aplicables en diferentes etapas del ciclo de vida ${ }^{(2)}$

El síndrome de Down constituye la primera causa genética de discapacidad intelectual. Se debe a una anomalía cromosómica que, en la mayoría de los casos, corresponde a una trisomía libre del cromosoma $21^{(4)}$. La prevalencia al nacimiento de esta entidad aumenta con la edad materna, siendo el riesgo aproximadamente de 1/1.500 a los 20 años, $1 / 900$ a los $30,1 / 350$ a los $35,1 / 100$ a los 40 y $1 / 25$ a los 45 años ${ }^{(5)}$. Durante el embarazo, existe la posibilidad de realizar la pesquisa del síndrome de Down a través de la evaluación combinada de la edad materna, la translucencia nucal, y los niveles en sangre materna de gonadotrofina coriónica humana y proteína plasmática $\mathrm{A}$ asociada al embarazo; entre las semanas 11 y 14 de gestación (pesquisa del $1^{0}$ trimestre) $)^{(6)}$. De acuerdo a los resultados de la pesquisa, o si existen otras indicaciones, puede recomendarse el diagnóstico prenatal a través de técnicas invasivas para determinar el cariotipo fetal (amniocentesis o biopsia corial).

Este síndrome se ha asociado tanto con menor peso al nacimiento ${ }^{(7,8)}$, como con parto pretérmino, en comparación con neonatos sin síndrome de Down ${ }^{(8)}$. Por otro lado, el parto pretérmino es una causa de bajo peso al nacer ${ }^{(9)}$, por lo que la edad gestacional debe tenerse en cuenta al analizar este último. También se ha asociado el síndrome de Down con la presencia de anomalías congénitas asociadas, siendo las más frecuentes las cardiopatías ${ }^{(10,11)}$. Estas comorbilidades, a su vez, han sido relacionadas con una disminución de la sobrevida de los afectados ${ }^{(8,12)}$. Ha sido descripto que cuando los niños nacen en maternidades de mayor complejidad, la mortalidad neonatal es menor ${ }^{(13)}$. Las maternidades de Argentina se clasifican según su complejidad en nivel 2, 3A y 3B, el 3B corresponde a los establecimientos con la complejidad más alta, con capacidad de atender a los recién nacidos de mayor riesgo, incluyendo a prematuros de menos de 1.500 gramos de peso, menos de 32 semanas de gestación, que requieren oxigenoterapia y asistencia respiratoria mecánica, alimentación parenteral y acceso a todas las especialidades pediátricas (entre ellas genetistas, cirujanos generales y cirujanos especialistas), y que cuentan con servicios de apoyo tales como diagnóstico por imágenes, hemoterapia y laboratorio clínico y bacteriológico, entre otros. En nuestro país, los niños con patología neonatal crítica y patologías quirúrgicas (incluyendo las del sistema nervioso central y las cardiológicas) deben ser atendidos en establecimientos con este nivel de complejidad ${ }^{(14)}$.

Algunas de las fuentes para estimar la prevalencia al nacimiento del síndrome de Down han sido los sistemas de vigilancia de anomalías congénitas, tales como el Estudio Colaborativo Latinoamericano de Malformaciones Congénitas (ECLAMC) ${ }^{(15)}$; la Agencia de Salud Pública de Canadá(16) , el consorcio International Clearinghouse for Birth Defects Surveillance and Research ${ }^{(17)}$, y la red Europea de Vigilancia de Anomalías Congénitas (EUROCAT) ${ }^{(18)}$, entre otros. En Argentina, la Red Nacional de Anomalías Congénitas (RENAC) Ileva a cabo desde el año 2009 la vigilancia de anomalías congénitas mayores (entre ellas síndrome de Down) en recién nacidos de las principales maternidades del territorio nacional ${ }^{(19)}$. En un estudio previo Campaña et al 
reportaron una prevalencia de 19,6 afectados cada 10.000 nacimientos en Argentina para los años 1994-2007(20); sin embargo, no se conocen investigaciones que se hayan realizado en nuestro país sobre su distribución por jurisdicciones o por edad materna, siendo esta última el principal factor de riesgo.

Los objetivos del presente estudio fueron determinar la prevalencia al nacimiento del síndrome de Down a nivel nacional y jurisdiccional en Argentina y según categorías de edad materna; la distribución de nacimientos con síndrome de Down por categorías de edad materna; la prevalencia y proporción de neonatos con síndrome de Down en quienes se realizó diagnóstico prenatal, según tipo de institución de nacimiento; y evaluar su asociación con el peso al nacer y edad gestacional al parto.

\section{POBLACIÓN Y MÉTODOS}

La fuente de datos fue la RENAC, el sistema de vigilancia de anomalías congénitas dependiente del Centro Nacional de Genética Médica, de la Administración Nacional de Laboratorios e Institutos de Salud "Dr. Carlos G. Malbrán", Ministerio de Salud de la Nación. Esta red incluye a las principales maternidades de todas las jurisdicciones del país, con una cobertura aproximada del $62 \%$ de los nacimientos del subsector público y del $43 \%$ del total de nacimientos. En la RENAC se reportan los recién nacidos con anomalías estructurales mayores, externas o internas, identificadas desde el nacimiento hasta el alta del hospital; las anomalías se describen en forma de redacción abierta y sin límite de anomalías por paciente. Se incluyen todos los recién nacidos vivos y los fetos muertos que pesan 500g o más. Las anomalías congénitas son codificadas por médicos genetistas de la coordinación de la RENAC, según la $10^{a}$ revisión de la Clasificación Internacional de Enfermedades con la adaptación del Royal College of Paediatrics and Child Health. Además, cada maternidad reporta el número de nacimientos totales producidos en cada hospital por mes ${ }^{(2,19,21)}$.
Para el presente estudio se incluyeron los casos con síndrome de Down (CIE-10: Q90), correspondientes al período comprendido entre noviembre de 2009 y diciembre de 2015. Se estimó la prevalencia ${ }^{(22)}$ en recién nacidos a nivel nacional y por jurisdicción, calculada como el número de casos con síndrome de Down reportados, dividido por el número de nacimientos totales de los hospitales que reportan a la RENAC. Para determinar si existe heterogeneidad en la prevalencia entre las jurisdicciones se llevó a cabo un metaanálisis de efectos aleatorios. Esta metodología permite ponderar el tamaño muestral de las diferentes jurisdicciones y compara dicha variabilidad con la variabilidad muestral esperada. Se calculó la prevalencia de afectados al nacimiento según grupos de edad materna y la distribución de nacimientos con y sin síndrome de Down en estos grupos. Para ello, se tomó de la Dirección de Estadísticas e Información en Salud (DEIS) el total de nacimientos por grupos de edad materna por año para el período $2009-2015^{(23)}$. Se comparó la prevalencia total de esta enfermedad entre subsectores (maternidades públicas vs. privadas/obra social) y según el nivel de complejidad de las maternidades públicas (nivel $3 \mathrm{~B} v$ s. nivel $3 \mathrm{~A}$ o 2$)^{(24)}$. Dicha comparación se realizó a través de las razones de prevalencia (RP); se utilizó un nivel de significación del 95\% según distribución de Poisson. Para la comparación entre subsectores, se realizó una regresión de Poisson usando como variable dependiente la prevalencia del síndrome de Down, como variable independiente el subsector, y la edad materna como variable de ajuste. Se calculó el porcentaje de casos con diagnóstico prenatal para el periodo 2013-2015 para cada subsector (maternidades públicas vs. privadas/obra social) y nivel de complejidad de las maternidades públicas (nivel 3B vs. nivel 3A o 2) y las RP; se utilizó un nivel de significación del 95\% según distribución binomial.

En el análisis del peso y la edad gestacional al nacimiento se usó, como grupo de comparación, los nacimientos reportados a la RENAC que no tenían anomalías congénitas mayores $(n=1.194)$. Para conocer la relación 
entre el síndrome de Down y el peso al nacimiento, se realizó una regresión lineal múltiple en la cual la variable dependiente fue el peso al nacimiento $y$, las variables independientes, el diagnóstico de síndrome de Down y la edad gestacional (variable de ajuste). Se utilizó la prueba de Mann-Whitney para determinar la diferencia en la distribución de la edad gestacional en los niños afectados y en aquellos sin anomalías congénitas mayores, previa evaluación de la normalidad de esta variable en ambas poblaciones con la prueba de asimetría y curtosis descripta por D'Agostino et al. ${ }^{(25)}$ y modificada por Royston ${ }^{(26)}$. Para el análisis estadístico se empleó el programa STATA/SE 13.

\section{RESULTADOS}

En un total de 1.358.158 nacimientos ocurridos en los hospitales participantes entre noviembre de 2009 y diciembre de 2015, se observaron 2.344 casos con síndrome de Down, de los cuales el 50,50\% fueron de sexo masculino. La prevalencia al nacimiento total fue de 17,26 por 10.000 nacimientos [IC95\% $(16,57-17,97)]$ y a nivel jurisdiccional varió entre 10,99 y 23,71 (Tabla 1).

El metaanálisis no mostró heterogeneidad entre las prevalencias jurisdiccionales: $Q$ de Cochrane $=16,86$; grados de libertad $=23$; $p=0,8 ; I^{2}=0,00$ (Figura 1). La prevalencia al

Tabla 1. Prevalencia del síndrome de Down al nacimiento, según jurisdicción. Argentina, 2009-2015.

\begin{tabular}{|c|c|c|c|c|}
\hline Jurisdicción & $\begin{array}{l}\text { Recién nacidos con } \\
\text { síndrome de Down }\end{array}$ & $\begin{array}{c}\text { Total de } \\
\text { nacimientos }\end{array}$ & $\begin{array}{c}\text { Prevalencia } \\
\text { (por } 10.000 \mathrm{NV} \text { ) }\end{array}$ & IC95\% \\
\hline Formosa & 23 & 20.930 & 10,99 & 6,$97 ; 16,49$ \\
\hline Corrientes & 28 & 24.683 & 11,34 & 7,$54 ; 16,4$ \\
\hline Entre Ríos & 38 & 30.304 & 12,54 & 8,$87 ; 17,21$ \\
\hline Santa Fe & 127 & 92.340 & 13,75 & 11,$47 ; 16,36$ \\
\hline Santiago del Estero & 54 & 35.488 & 15,22 & 11,$43 ; 19,85$ \\
\hline La Rioja & 21 & 13.524 & 15,53 & 9,$61 ; 23,74$ \\
\hline Rio Negro & 17 & 10.712 & 15,87 & 9,$24 ; 25,41$ \\
\hline Córdoba & 115 & 71.234 & 16,14 & 13,$33 ; 19,38$ \\
\hline Chaco & 80 & 49.385 & 16,20 & 12,$85 ; 20,16$ \\
\hline Buenos Aires & 661 & 407.450 & 16,22 & 15,$01 ; 17,51$ \\
\hline Santa Cruz & 10 & 6.163 & 16,23 & 7,$78 ; 29,84$ \\
\hline La Pampa & 15 & 9.130 & 16,43 & 9,$2 ; 27,1$ \\
\hline Salta & 108 & 65.371 & 16,52 & 13,$55 ; 19,95$ \\
\hline San Luis & 31 & 16.906 & 18,34 & 12,$46 ; 26,03$ \\
\hline Jujuy & 58 & 30.834 & 18,81 & 14,$28 ; 24,32$ \\
\hline Tucumán & 161 & 85.172 & 18,90 & 16,$1 ; 22,06$ \\
\hline San Juan & 69 & 35.634 & 19,36 & 15,$07 ; 24,51$ \\
\hline Mendoza & 119 & 59.072 & 20,14 & 16,$69 ; 24,11$ \\
\hline CABA & 374 & 184.382 & 20,28 & 18,$28 ; 22,45$ \\
\hline Chubut & 29 & 14.270 & 20,32 & 13,$61 ; 29,19$ \\
\hline Misiones & 113 & 54.904 & 20,58 & 16,$96 ; 24,74$ \\
\hline Neuquén & 47 & 20.707 & 22,70 & 16,$68 ; 30,18$ \\
\hline Tierra del Fuego & 12 & 5.225 & 22,97 & 11,$87 ; 40,12$ \\
\hline Catamarca & 34 & 14.338 & 23,71 & 16,$42 ; 33,14$ \\
\hline Total & 2.344 & 1.358 .158 & 17,26 & 16,$57 ; 17,97$ \\
\hline
\end{tabular}




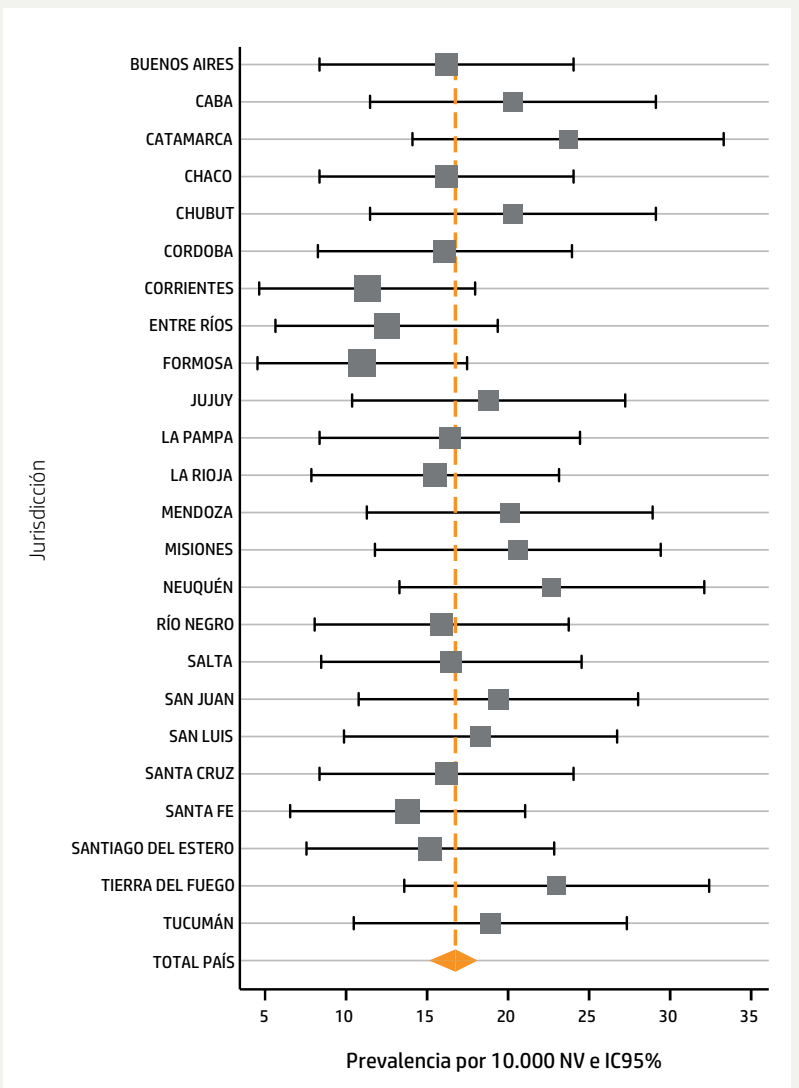

Figura 1. Metaanálisis de la prevalencia del síndrome de Down al nacimiento, según jurisdicción y total país. Argentina, 2009-2015.

Fuente: Elaboración propia basada en datos de la Red Nacional de Anomalías Congénitas (RENAC) (21).

IC95\%= Intervalo de confianza del 95\%. nacimiento de síndrome de Down aumentó a medida que la edad materna fue mayor y fue significativamente más alta a partir de los 35 años (Figura 2).

Se comparó la distribución porcentual del total de los nacidos con y sin síndrome de Down según categorías etarias, y se observó que el mayor porcentaje de los nacidos sin síndrome de Down corresponde al grupo de edad materna de 20 a 24 años y, el mayor porcentaje de los nacidos con síndrome de Down, a mujeres con edad mayor o igual a 35 años (Figura 3). Las maternidades privadas/ obra social presentaron una prevalencia significativamente mayor que las maternidades públicas; y dentro del subsector público, las maternidades de mayor complejidad fueron las que presentaron una prevalencia significativamente mayor (Tabla 2).

La prevalencia también fue mayor en el subsector privado que en el público de acuerdo a la regresión de Poisson $[R P=1,27$; IC95\% $(1,10 ; 1,47)]$. Sin embargo, al ajustar por edad materna, el efecto del subsector no se observó $[R P=0,99$; IC 95\% $(0,79-1,25)]$. El porcentaje de afectados con diagnóstico prenatal fue significativamente mayor en las maternidades privadas/obra social, así como también fue significativamente mayor en las maternidades públicas de nivel 3B (mayor complejidad) en comparación con las maternidades públicas de nivel 3A o 2 (menor complejidad) (Tabla 3).

La regresión lineal mostró que el peso al nacer de los niños con síndrome de Down, ajustado por edad gestacional, fue 294,78 gramos menor que los no afectados [coeficiente $\beta$ síndrome de Down = -294,78; IC95\% (-332,02; -257,54)]; [coeficiente $\beta$ edad gestacional $=169,4 ;$ IC95\% $(163,05 ; 175,86)$; $\left.p<0,001 ; R^{2} 0,45\right]$. La prueba de asimetría y curtosis evidenció que la distribución de la edad gestacional no es normal, tanto en los nacidos con síndrome de Down $(p<0,01)$

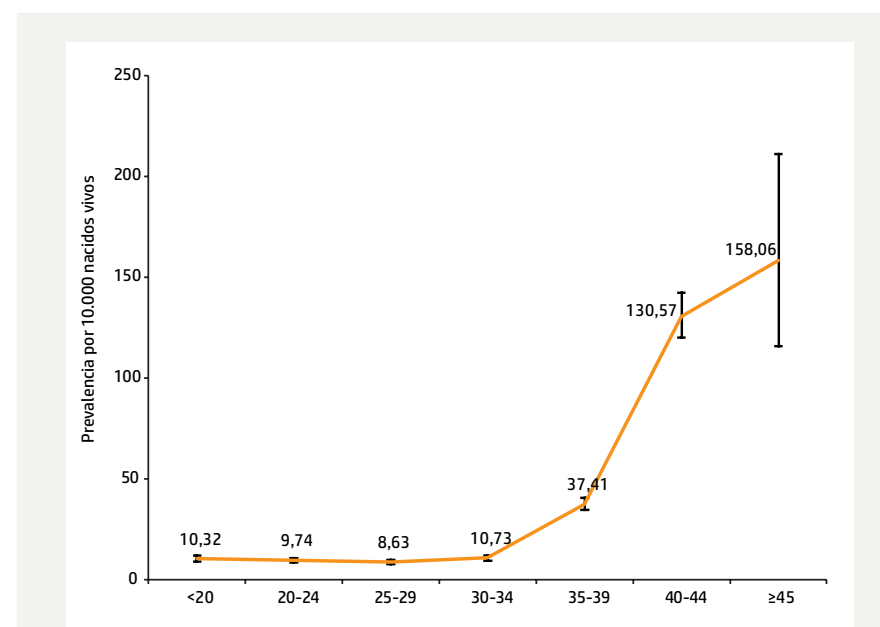

Figura 2. Prevalencia del síndrome de Down, según grupos de edad materna. Argentina, 2009-2015.

Fuente: Elaboración propia basada en datos de la Red Nacional de Anomalías Congénitas $(\text { RENAC) })^{(21)}$ y Dirección de Estadísticas e Información en Salud ${ }^{(23)}$. 


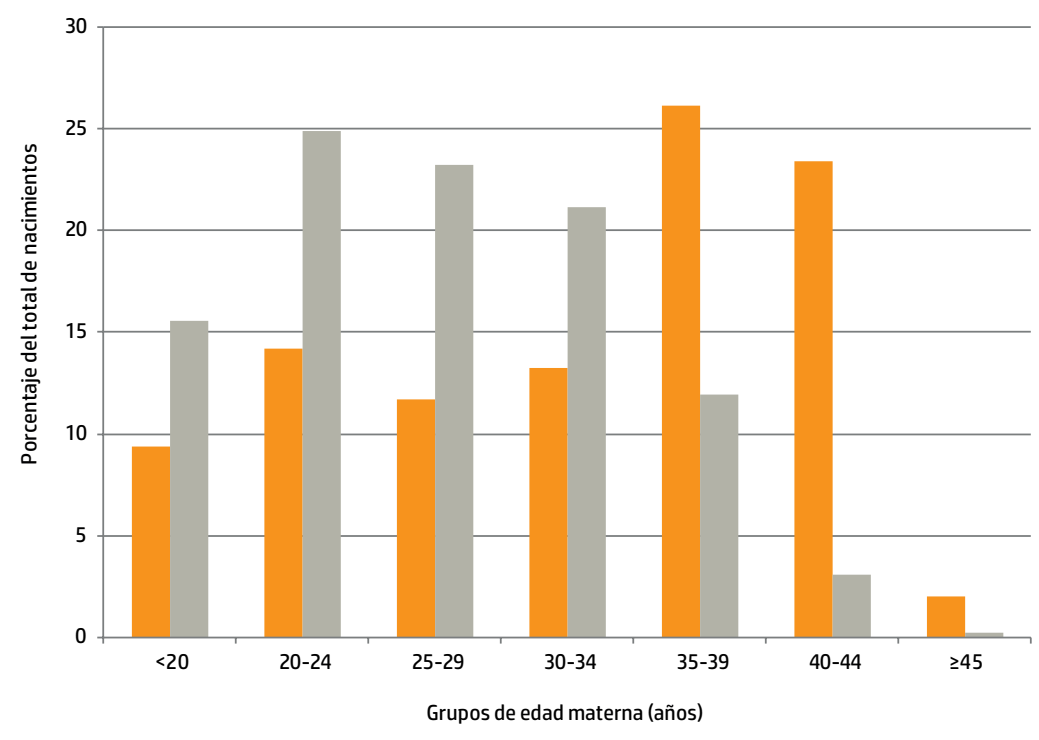

Recién nacidos con síndrome de Down

Recién nacidos sin síndrome de Down

Figura 3. Distribución porcentual, según grupos de edad materna, de los recién nacidos con síndrome de Down y sin síndrome de Down. Argentina, 2009-2015.

Fuente: Elaboración propia basada en datos de la Red Nacional de Anomalías Congénitas (RENAC)(21) y Dirección de Estadisticas e Información en Salud ${ }^{(23)}$.

Tabla 2. Prevalencia y razón de prevalencia de síndrome de Down, según el subsector y el nivel de complejidad de las maternidades. Argentina, 2009-2015.

\begin{tabular}{|c|c|c|c|c|c|}
\hline Clasificación de las maternidades & $\mathrm{n}$ & $\begin{array}{c}\text { Prevalencia } \\
\text { (por } 10.000 \mathrm{NV} \text { ) }\end{array}$ & IC95\% & $\mathrm{RP}$ & IC95\% \\
\hline Subsector privado/obra social & 203 & 21,55 & 18,$69 ; 24,72$ & $1,33^{*}$ & 1,$27 ; 1,38$ \\
\hline Subsector público ${ }^{1}$ & 2.141 & 16,25 & 15,$40 ; 17,14$ & - & - \\
\hline Subsector público, nivel de complejidad 3B & 1.394 & 17,45 & 16,$29 ; 18,66$ & $1,19^{*}$ & 1,$14 ; 1,25$ \\
\hline Subsector público, nivel de complejidad $3 \mathrm{~A}$ y $2^{1}$ & 747 & 14,62 & 13,$38 ; 15,92$ & - & - \\
\hline
\end{tabular}

Tabla 3. Porcentaje de casos de síndrome de Down con diagnóstico prenatal y razón de prevalencia, según el subsector y el nivel de complejidad de las maternidades. Argentina, 2013-2015.

\begin{tabular}{|c|c|c|c|c|c|}
\hline Clasificación de las maternidades & $\mathrm{n}$ & $\begin{array}{l}\text { Diagnóstico } \\
\text { prenatal (\%) }\end{array}$ & IC95\% & $\mathrm{RP}$ & IC95\% \\
\hline Subsector privado/obra social & 62 & 30,54 & 24,$28 ; 37,37$ & $2,16^{*}$ & 2,$08 ; 2,24$ \\
\hline Subsector público ${ }^{1}$ & 193 & 14,11 & 12,$30 ; 16,06$ & - & - \\
\hline Subsector público, nivel de complejidad 3B & 134 & 15,82 & 13,$42 ; 18,45$ & $1,40^{*}$ & 1,$32 ; 1,46$ \\
\hline Subsector público, nivel de complejidad $3 \mathrm{~A}$ y $2^{1}$ & 59 & 11,32 & 8,$59 ; 14,15$ & - & - \\
\hline
\end{tabular}


como en los nacimientos sin anomalías mayores $(p<0,01)$. La mediana de edad gestacional de estos dos grupos fue de 39 semanas, y la prueba de Mann-Whitney mostró que la distribución de la edad gestacional en ambos grupos no es igual $(p=0,02)$.

\section{DISCUSIÓN}

La prevalencia del síndrome de Down al nacimiento, de 17,26/10.000, observada en este estudio, es menor a la reportada previamente para Argentina entre 1994 y 2007 por Campaña et al., que fue de 19,6/10.000 ${ }^{(20)}$. Dicho trabajo fue elaborado por el grupo ECLAMC con datos provenientes de un número menor de hospitales, pero de mayor complejidad; en cambio la RENAC cubre un mayor número de maternidades del sector público, incluyendo hospitales de distinto nivel de complejidad. La diferencia observada puede deberse al mayor sesgo de derivación en el ECLAMC. Este sesgo consiste en que, si hubo diagnóstico prenatal, las mujeres embarazadas fueron derivadas para su atención y la del recién nacido a hospitales de mayor complejidad, lo cual genera un aumento de la prevalencia hospitalaria. Sin embargo, la prevalencia en nuestro estudio es similar a la reportada por el grupo ECLAMC para América del Sur en el año 2012 que fue de 17,85/10.000 (15) con cobertura de hospitales del sector público y privado de distintos países del continente, con variados niveles de complejidad por lo que probablemente el sesgo de derivación en este estudio sea menor.

Debe considerarse la posibilidad de acceso al diagnóstico prenatal y posterior terminación electiva del embarazo debido a anomalías fetales para interpretar las diferencias de prevalencia con otros países. Por ejemplo, en Canadá, la Agencia de Salud Pública reportó una prevalencia de 14,1/10.000 entre los años 1998 y $2007^{(16)}$. En este reporte no se incluyen las terminaciones electivas del embarazo por anomalía fetal, práctica permitida en ese país, lo que justifica la menor prevalencia en nacidos vivos. La red
Europea de Vigilancia de Anomalías Congénitas (EUROCAT) publicó una prevalencia de síndrome de Down de 10,34/10.000 en recién nacidos y fetos muertos para el período 2011-2015, la cual aumenta a 23,88/10.000 al incluir las terminaciones electivas del embarazo por anomalía fetal ${ }^{(18)}$. En el año 2012 se registró en el estado de Australia Occidental una prevalencia total del síndrome de Down de 28,74/10.000 considerando terminaciones electivas (que corresponden al $73 \%$ de los embarazos con síndrome de Down) y de 7,33/10.000 en nacidos vivos ${ }^{(15)}$. En Noruega, en 2011, se registró una prevalencia de 22,55/10.000, incluyendo terminaciones electivas $(34,7 \%$ de los embarazos con síndrome de Down) y de $13,9 / 10.000$ en nacidos vivos ${ }^{(27)}$.

En nuestro estudio, la prevalencia por jurisdicciones mostró variaciones regionales, pero de acuerdo a los resultados del metaanálisis se concluyó que no existe heterogeneidad estadísticamente significativa.

Al analizar la prevalencia del síndrome de Down según segmentos de edad materna se evidencia que esta se mantiene relativamente estable para edades menores de 35 años, con un aumento a partir de esta edad, el cual es más pronunciado a partir de los 40 años, estos resultados son coincidentes con los previamente observados en otros estudios $^{(5,28,29)}$. Comparando la distribución de nacimientos de niños con síndrome de Down y sin síndrome de Down según grupos de edad materna, se observó que el 48,5\% de los niños con síndrome de Down nacen de madres menores de 35 años, en quienes ocurre el $85 \%$ de todos los nacimientos ${ }^{(23)} y$, el 51,5\% restante, en mujeres de 35 años o más, grupo de edad en el que se produce el $15,0 \%$ del total de nacimientos del país. Ha sido referido que los embarazos no deseados son especialmente frecuentes en adolescentes, mujeres solteras y mayores de $40 \mathrm{años}^{(30)}$. Este último grupo, si bien representa una proporción minoritaria del total de embarazos, da cuenta de aproximadamente el $25 \%$ de los casos de síndrome de Down. Medidas de prevención orientadas a disminuir los embarazos no buscados en este grupo de riesgo, 
posiblemente representen una medida adecuada de salud pública.

El subsector del sistema de salud en el que las pacientes reciben atención médica puede tomarse como un indicador indirecto del nivel socioeconómico, siendo más frecuente que las mujeres de mayor nivel socioeconómico sean atendidas en el ámbito privado/obra social. La prevalencia de síndrome de Down en el subsector público fue menor a la hallada en el privado/obra social. Se evidenció que esto se debe a una mayor edad materna en el subsector privado/obra social. Esta observación concuerda con lo reportado por otros autores: la edad materna es mayor en mujeres con un nivel socioeconómico más alto(31,32). La mayor prevalencia y el mayor porcentaje de diagnóstico prenatal en maternidades nivel 3B (mayor complejidad) sería atribuible a la derivación de las mujeres embarazadas con diagnóstico prenatal patológico hacia establecimientos de mayor nivel de complejidad. Asimismo, el diagnóstico prenatal es 2,16 veces mayor en los nacimientos de establecimientos privados que en los públicos, lo cual podría explicarse por el mayor acceso a controles obstétricos y a métodos de diagnóstico prenatal invasivo en el ámbito privado, un posible reflejo de la existencia de barreras de tipo culturales, geográficas y económicas para estos estudios en nuestro país. En un estudio previo en una cohorte de mujeres sudamericanas, entre ellas argentinas, Campaña et al. reportaron que las mujeres atendidas en el subsector privado tenían un mayor número de consultas y ecografías prenatales que las del sector público ${ }^{(32)}$. Dado el perfil de morbimortalidad de los recién nacidos con síndrome de Down, se considera que deben nacer en una maternidad con la máxima complejidad. Por este motivo cobra especial importancia la posibilidad de realizar el diagnóstico prenatal para la planificación del nacimiento en un establecimiento sanitario que cuente con la complejidad adecuada, así como para la preparación del equipo de salud y del grupo familiar para recibir al recién nacido.

El hallazgo de un peso de nacimiento menor concuerda con lo descripto previa- mente $^{(7,8)}$. Gorlin et al. describen un peso al nacer de 400 gramos menor en pacientes con síndrome de Down que en niños sanos ${ }^{(33)}$. En nuestro estudio, se evidenció que la mediana de edad gestacional al nacimiento en niños con síndrome de Down no difiere de la correspondiente a niños sin anomalías mayores, aunque la distribución de esta variable no es igual en estos dos grupos. Ello podría deberse a que en los niños con síndrome de Down se registren valores menores de edad gestacional, como ha sido reportado por otros autores ${ }^{(8)}$.

\section{CONCLUSIONES}

El síndrome de Down es una de las anomalías congénitas más frecuentes en nuestro país. Puede ser detectada prenatalmente $y$, considerando las comorbilidades que presenta, requiere de la participación de un equipo interdisciplinario para el seguimiento de los niños y sus familias. Resulta fundamental, en estos casos, la educación y la planificación del embarazo con un adecuado asesoramiento preconcepcional, en especial, en casos de mujeres con edad materna avanzada.

Una fortaleza del presente estudio es que incluyó a los recién nacidos de las principales maternidades del sector público de las 24 jurisdicciones del país, con una alta cobertura de nacimientos y distintos niveles de complejidad. Entre las limitaciones del estudio, cabe destacar que, con respecto al peso al nacer, no se cuenta con otros datos que puedan influir en esta variable, tales como enfermedades maternas durante el embarazo. Otra debilidad del estudio fue que la variable diagnóstico prenatal se reportó de forma sistemática solo a partir del año 2013, por lo que no se incluyen periodos anteriores.

El conocimiento de las características epidemiológicas de esta entidad permite la implementación de políticas sanitarias específicas para este grupo de pacientes. Los países en los que está permitida la terminación del embarazo por anomalías fetales presentan una prevalencia de síndrome de Down en 
nacidos vivos sustancialmente menor. El acceso a la detección prenatal y terminación electiva del embarazo por anomalía fetal, junto con las diferencias en la estructura de edad materna de la población, representan los principales determinantes de la prevalencia de recién nacidos con síndrome de Down.

\section{AGRADECIMIENTOS}

A todos los profesionales que forman parte de la Red Nacional de Anomalías Congénitas en las 24 jurisdicciones del país.

\section{REFERENCIAS BIBLIOGRÁFICAS}

1. Rimoin DL, Pyeritz RE, Korf BR. Emery \& Rimoin's principles and practice of medical genetics. 6a ed. Cambridge: Academic Press; 2013.

2. Groisman B, Bidondo MP, Barbero P, Gilib JA, Liascovich R, Grupo de Trabajo RENAC. RENAC: Registro Nacional de Anomalías Congénitas de Argentina. Archivos Argentinos de Pediatría. 2013; 111(6):484-494.

3. Dirección de Estadísticas e Información en Salud. Defunciones de menores de 5 años por grupo de edad según causas seleccionadas [Internet]. Buenos Aires: DEIS; 2016 [citado 8 abr 2018]. Disponible en: https://tinyurl.com/ yxtk2rz9.
4. Nussbaum RL, Mclnnes RR, Willard HF. Thompson \& Thompson genetics in medicine. 8a ed. Canada: Elsevier; 2016.

5. Snijders RJ, Sundberg K, Holzgreve W, Henry G, Nicolaides KH. Maternal age- and gestationspecific risk for trisomy 21. Ultrasound in Obstetrics \& Gynecology. 1999;13:167-170.

6. Wright D, Spencer K, Kagan KK y col. First-trimester combined screening for trisomy 21 at 7-14 weeks' gestation. Ultrasound in Obstetrics \& Gynecology. 2010;36:404-411.

7. Leonard S, Bower C, Petterson B, Leonard H. Survival of infants born with Down's syndrome: 1980-96. Paediatric and Perinatal Epidemiology. 2000;14:163-171.

8. Glasson EJ, Jacques A, Wong K, Bourke J, Leonard $\mathrm{H}$. Improved survival in Down syndrome over the last 60 years and the impact of perinatal factors in recent decades. Journal of Pediatrics. 2016;169:214-220. doi: 10.1016/j.jpeds.2015.10. 083. 
9. Kliegman RM, Behrman RE, Jenson HB, Stanton BF. Nelson: Tratado de pediatría. 18va ed. Barcelona: Elsevier; 2008.

10. Cleves MA, Hobbs CA, Cleves PA, Tilford JM, Bird TM, Robbins JM. Congenital defects among liveborn infants with Down syndrome. Birth Defects Research (Part A). 2007;79:657-663. doi: 10.1002/bdra.20393.

11. Morris JK, Garne E, Wellesley D, Addor MC, Arriola L, Barisic I, Beres J, Bianchi F, Budd J, Dias CM, et al. Major congenital anomalies in babies born with Down syndrome: A EUROCAT population-based registry study. American Journal of Medical Genetics Part A. 2014;9999:1-8. doi: 10.1002/ajmg.a.36780.

12. Goldman SE, Urbano RC, Hodapp RM. Determining the amount, timing and causes of mortality among infants with Down syndrome. Journal of Intellectual Disability Research. 2011;55:85-94. doi: 10.1111/j.1365-2788.2010.01349.x.

13. Berg CJ, Druschel CM, McCarthy BJ, LaVoie M, Floyd RL. Neonatal mortality in normal birth weight babies: does the level of hospital care make a difference? American Journal of Obstetrics Gynecology. 1989;161(1):86-91. doi: 10.1016/00029378(89)90239-1.

14. Ministerio de Salud de la Nación Argentina. Resolución 641/2012: Directriz de organización y funcionamiento de los servicios de cuidados neonatales [Internet]. Buenos Aires: Ministerio de Justicia y Derechos Humanos de la Nación; 2012 [citado 12 jul 2017]. Disponible en: https://tinyurl. com/y436j5em.

15. International Clearinghouse for Birth Defects Surveillance and Research. Annual Report 2014 [Internet]. Roma: ICBDRS; 2014 [citado 12 jul 2017]. Disponible en: https://tinyurl.com/y4fus4ox.

16. Public Health Agency of Canada. Congenital anomalies in Canada 2013: A perinatal health surveillance report [Internet]. Ottawa: Public Health Agency of Canada; 2013 [citado 14 jul 2017]. Disponible en: https://tinyurl.com/y5jkb9lk.

17. Leoncini E, Botto LD, Cocchi G, Annerén G, Bower C, Halliday J, Amar E, Bakker MK, Bianca S, Canessa Tapia MA, et al. How valid are the rates of Down syndrome internationally?: Findings from the International Clearinghouse for Birth Defects Surveillance and Research. American Journal of Medical Genetics Part A. 2010;152A(7):16701680. doi: 10.1002/ajmg.a.33493.

18. EUROCAT prevalence tables. Cases and prevalence of Down syndrome from 2011-2015 [citado 14 jul 2017]. Disponible en: https://tinyurl. com/yypz69we.

19. Bidondo MP, Groisman B, Gili JA, Liascovich R, Barbero P. Estudio de prevalencia y letalidad neonatal en pacientes con anomalías congénitas seleccionadas con datos del Registro Nacional de Anomalías Congénitas de Argentina. Archivos Argentinos de Pediatría. 2015;113(4):295-302.

20. Campaña H, Pawluk MS, López Camelo JS. Prevalencia al nacimiento de 27 anomalías congénitas seleccionadas, en 7 regiones geográficas de la Argentina. Archivos Argentinos de Pediatría. 2010;108(5):409-417.

21. Groisman B, Duarte S, Gili J, Liascovich R, Camelo L, Santiago J, Grupo de Trabajo RENAC. Registro Nacional de Anomalías Congénitas de Argentina, Reporte anual RENAC 2016: Análisis epidemiológico de las anomalías congénitas en recién nacidos registradas en el año 2015 en la República Argentina. Buenos Aires: Ministerio de Salud de la Nación; 2016.

22. Mason CA, Kirby RS, Sever LE. Prevalence is the preferred measure of frequency of birth defects. Birth Defects Research Part A. 2005;73(10):690-692.

23. Dirección de Estadísticas e Información en Salud. Estadísticas vitales [Internet]. Buenos Aires: Ministerio de Salud de la Nación [citado 12 jul 2017]. Disponible en: https://tinyurl.com/y2bftfyw.

24. Dirección Nacional de Maternidad, Infancia y Adolescencia. Mapa sanitario [Internet]. Buenos Aires: Ministerio de Salud de la Nación [citado 7 feb 2018]. Disponible en: http://datos.dinami.gov. ar/produccion/cuadromando.

25. D'Agostino RB, Belanger A, D'Agostino RB Jr. A suggestion for using powerful and informative tests of normality. The American Statistician. 1990; 44(4):316-321.

26. Royston P. Comment on sg3.4 and an improved D'Agostino test. Stata Technical Bulletin. 1992; 1(3):23-24.

27. International Clearinghouse for Birth Defects Surveillance and Research. Annual Report 2013 [Internet]. Roma: ICBDRS centre; 2013 [citado 19 mar 2018]. Disponible en: https://tinyurl.com/ y58sag3j.

28. Cuckle HS, Wald NJ, Thompson SG. Estimating a woman's risk of having a pregnancy associated with Down's syndrome using her age and serum alpha-fetoprotein level. British Journal of Obstetrics and Gynaecology. 1987;94(5):387-402. 
29. Morris JK, Mutton DE, Alberman E. Revised estimates of the maternal age specific live birth prevalence of Down's syndrome. Journal of Medical Screening. 2002;9(1):2-6.

30. Langer A. Unwanted pregnancy: impact on health and society in Latin America and the Caribbean. Revista Panamericana de Salud Pública. 2002;11(3):192-204.

31. Goisis A, Schneider D, Myrskylä M. Secular changes in the association between advanced maternal age and the risk of low birth weight: a cross-cohort comparison in the UK. Population
Studies. 2018;27:1-17. doi: 10.1080/00324728. 2018.1442584.

32. Campaña H, Ermini $M$, Aiello HA, Krupitzki H, Castilla EE, López-Camelo JS, Latin American Collaborative Study of Congenital Malformations Study Group. Prenatal sonographic detection of birth defects in 18 hospitals from South America. Journal of Ultrasound in Medicine. 2010;29(2):203-212.

33. Gorlin RJ, Cohen MM, Hennekam RCM. Syndromes of the head and neck. 4a ed. New York: Oxford University Press; 2001.

\section{FORMA DE CITAR}

Martini J, Bidondo MP, Duarte S, Liascovich R, Barbero P, Boris Groisman. Prevalencia del síndrome de Down al nacimiento en Argentina. Salud Colectiva. 2019;15:e1863. doi: 10.18294/sc.2019.1863.

Recibido: 3 de mayo de 2018 | Versión final: 2 de abril de 2019 | Aprobado: 4 de junio de 2019

Esta obra está bajo una licencia de Creative Commons Reconocimiento-NoComercial 4.0 Internacional. Reconocimiento - Permite copiar, distribuir y comunicar públicamente la obra. A cambio, se debe reconocer y citar al autor original. No Comercial - Esta obra no puede ser utilizada con finalidades comerciales, a menos que se obtenga el permiso. 